\title{
RAPID TURN-TAKING AS A CONSTANT FEATURE OF SIGNED CONVERSATIONS
}

\author{
CONNIE DE VOS $1^{* 1}$ \\ ${ }^{*}$ Corresponding Author: c.devos@ let.ru.nl \\ ${ }^{1}$ Center for Language Studies, Radboud University, Nijmegen, the Netherlands
}

Social interaction is the primary ecological niche for languages to evolve and to emerge (Levinson 2006). The spontaneous conversations that characterize our every-day interactions are remarkably rapid across a wide range of typologically diverse spoken languages with most turns timed $\sim 200 \mathrm{~ms}$ after the prior (Stivers et al. 2009). Corpus analysis of the Sign Language of the Netherlands (NGT) has shown that this main observation also holds in the visual-gestural language modality, when the different movement phases of the hands are taken into account (de Vos et al. 2015). The present study is the first to investigate whether turntiming is a constant pressure of conversation in language emergence by looking at different generations of the emergent signing variety Kata Kolok.

Kata Kolok is a signed language that has emerged outside the influence of any other signed languages. It has been used by six subsequent generations of native signers in a village community of Bali. On a par with the Stivers et al. comparative study, I collected question-answer sequences from the Kata Kolok Corpus (de Vos 2016), which covers generations III-V of adult signers in dyadic and triadic settings, as well as the NGT Interactive Corpus (de Vos et al. 2015). Prior work on sign languages shows that signed turns at talk can be effectively delineated on the basis of the lexically-specified movement phases of signs as well as prosodic turn-boundary markers such as eye blinks and nods. Each question-answer sequence was therefore transcribed for their movement phases (preparation, stroke, hold, retraction) as well as prosodic cues marking questionhood and phrase boundaries (e.g. blinks, raised eyebrows, nods) (cf. Kita et al. 1999). Turn-timing was calculated stroke-to-stroke, i.e. as the floor transition offset (FTO) between the end of the question's turn-final stroke and the start of the answer's turn-initial stroke (cf. de Vos et al. 2015). I report on both overall means for stroke-to-stroke turn transitions as well as the "Optimal turntiming" measure, which subtracts the cross-linguistic default of 200ms off the original FTO and collapses them into an absolute value to assess how effective 
turn transitions are across generations as well as both language communities. Linear mixed effects modelling in $\mathrm{R}$ reveals there are no significant effects of either language community or generation, but does indicate that NGT signers are faster in triadic settings when allowing random effects for recording session and signer. This is illustrated by Figure 1. below.

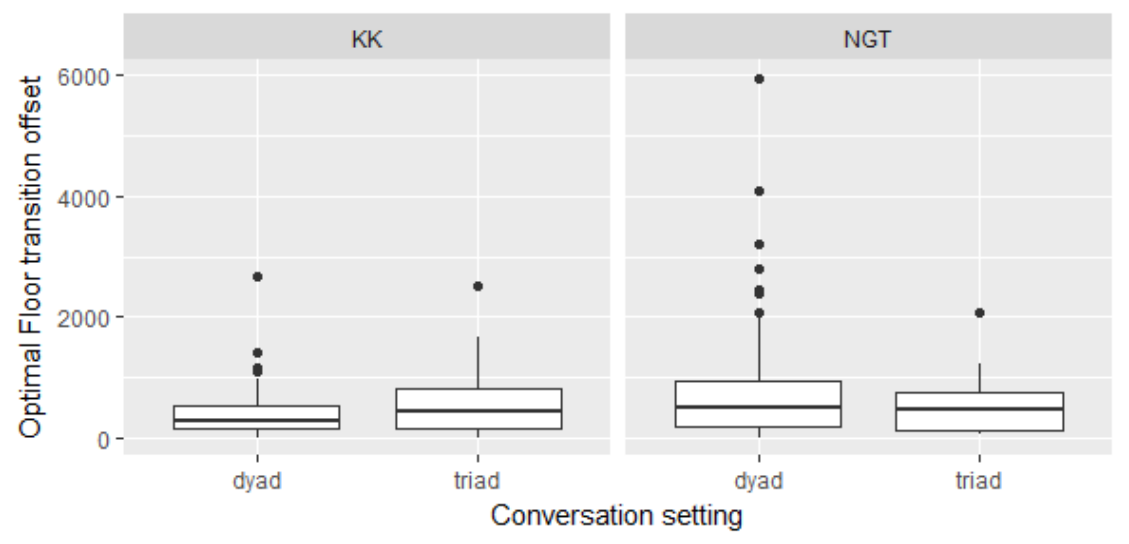

Figure 1. Floor transition offsets in Kata Kolok and Sign Language of the Netherlands (NGT)

The present results further extend the generalization that signed languages are within the cross-linguistic range that can be expected on the basis of spoken language turn-timing, by including data from the sign language isolate Kata Kolok (cf. Stivers et al. 2009). Moreover, these results hold across generations suggesting that rapid turn-timing might a stable aspect of conversation in language emergence. The fact that NGT signers take turns more efficiently in triadic settings is consistent with the view that rapid turn-taking exerts a time pressure on both language producers and perceivers in such settings.

Prior work has shown that prosodic cues (e.g. of questionhood) are crucial in the anticipation of turn-endings (Casillas et al. 2015). Moreover, such prosodic signaling appears to be unstable in the early stages of sign language emergence (Sandler et al. 2011). Future work therefore focuses on the hypothesis that the time-pressure of real-life turn-taking may lead to the optimization of the prosodic features of utterances during sign language emergence. 


\section{Acknowledgements}

This work was supported by the NWO VENI grant 'The face in sign language interaction' awarded to Connie de Vos.

\section{References}

Casillas, M., De Vos, C., Crasborn, O., \& Levinson, S. C. (2015). The perception of stroke-to-stroke turn boundaries in signed conversation. In D. C. Noelle, R. Dale, A. S. Warlaumont, J. Yoshimi, T. Matlock, C. D. Jennings, \& P. R. Maglio (Eds.), Proceedings of the 37th Annual Meeting of the Cognitive Science Society (CogSci 2015) (pp. 315320). Austin, TX: Cognitive Science Society.

De Vos, C. (2016). Sampling shared sign languages. Sign Language Studies, 16(2), 204-226.

De Vos, C., Torreira, F., \& Levinson, S. C. (2015). Turn-timing in signed conversations: Coordinating stroke-to-stroke turn boundaries. Frontiers in Psychology, 6: 268.

Kita, S., Van Gijn, I., and van der Hulst, H. (1998). "Movement phases in signs and co-speech gestures, and their transcription by human coders," in Gesture and Sign Language in Human-Computer Interaction, eds I. Wachsmuth and M. Fröhlich (Berlin: Springer), 23-35.

Levinson, S. C. (2006). "On the human interaction engine," in Roots of Human Sociality: Culture, Cognition and Interaction, eds N. J. Enfield and S. C. Levinson (Oxford: Berg), 39-69.

Sandler, W., Meir, I., Dachkovsky, S., Padden, C., \& Aronoff, M. (2011). The emergence of complexity in prosody and syntax. Lingua, 121(13), 2014-2033.

Stivers, T., Enfield, N. J., Brown, P., Englert, C., Hayashi, M., Heinemann, T.,et al. (2009). Universals and cultural variation in turn-taking in conversation. Proc. Natl. Acad. Sci. U.S.A. 106, 10587-10592. doi: 10.1073/pnas.0903616106 